\title{
The response of a floating ice sheet to an accelerating line load
}

\author{
By J. MILES ${ }^{1}$ AND A. D. SNEY D ${ }^{2}$ \\ ${ }^{1}$ Cecil H. and Ida M. Green Institute of Geophysics and Planetary Physics, University of California, \\ San Diego, La Jolla, CA 92093-0225, USA \\ ${ }^{2}$ University of Waikato, Private Bag 3105, Hamilton, New Zealand
}

(Received 12 June 2003 and in revised form 28 August 2003)

The two-dimensional response of a thin, floating sheet of ice to a line load that accelerates from rest at $t=0$ to a uniform velocity $V$ for $t \geqslant T$ is determined through an integral-transform solution of the linearized equations of motion. If $T=0-$ i.e. if the load is impulsively started with velocity $V$ - the solution exhibits singularities at $V=c_{0}$, the shallow-water-gravity-wave speed, and $V=c_{\min }$, the minimum speed for transverse motion of the ice, but these singularities are avoided by the acceleration of the load through the critical speeds.

\section{Introduction}

The problem of a load moving with a uniform velocity $V$ over a sheet of ice floating on a large body of water has been studied both analytically and experimentally; see Squire et al. (1996) and the references given therein. The analytical solution is characterized by singularities associated with $V=c_{0}$, the shallow-water-gravitywave speed, and $V=c_{\min }$, the minimum speed for transverse wave motion of the ice. These singularities may be avoided through the incorporation of either dissipation (Squire et al. 1996, §§4.2.2, 5.6) or nonlinearity (Pǎrǎu \& Dias 2002), but it appears to have been overlooked that they also can be avoided by allowing for acceleration of the load through the critical speeds, Miles (1960).

Against this background, we consider here the wave motion induced by the line load

$$
p(x, t)=F \delta[x-X(t)]
$$

and posit the resulting transverse displacement of the ice in the form

$$
\eta(x, t)=\int_{0}^{t} G[x-X(\tau), t-\tau] \mathrm{d} \tau,
$$

where $\delta$ is Dirac's delta function, $G$ is a Green's function (see $\S 2$ ), and

$$
X(t)=\left\{\begin{array}{l}
X_{A}(T)+V(t-T) \quad(t \geqslant T) \\
X_{A}(t) \quad(0 \leqslant t \leqslant T),
\end{array}\right.
$$

which describes an accelerated motion $X_{A}(t)$ from rest at $t=0$ to a uniform velocity $V$ for $t \geqslant T$. $\left(X_{A}(t)=\frac{1}{2} A t^{2}\right.$ for a uniform acceleration $A$.) It then follows from the linearity of the boundary-value problem for $\eta$ that

$$
\eta(x, t)=\left\{\begin{array}{l}
\eta_{A}(x, T)+\eta_{V}[x-X(T), t-T] \quad(t>T) \\
\eta_{A}(x, t) \quad(0 \leqslant t \leqslant T),
\end{array}\right.
$$


where: $\eta_{A}$ is given by (1.2) with $X(t)=X_{A}(t) ; \eta_{V}$ is given by (1.2) with $X(t)=V t$ and describes the motion associated with an abruptly imposed velocity $V$.

\section{Boundary-value problem}

The two-dimensional, linearized boundary-value problem for the determination of the velocity potential $\phi(x, y, t)$ and the displacement $\eta(x, t)$ of a thin (negligible inertia) sheet of ice that overlies a body of water of depth $H$ is described by Laplace's equation

$$
\phi_{x x}+\phi_{y y}=0 \quad(-\infty<x<\infty,-H<y<0),
$$

the kinematic boundary conditions

$$
\phi_{y}=0 \quad(y=-H), \quad \phi_{y}=\eta_{t} \quad(y=0),
$$

the initial conditions

$$
\phi=\eta=0 \quad(t=0)
$$

and the dynamical boundary condition

$$
\rho\left(\phi_{t}+g \eta\right)+D \partial_{x}^{4} \eta=-p(x, t) \quad(y=0),
$$

where $\rho$ is the density of the fluid, $-\rho\left(\phi_{t}+g \eta\right)$ is the fluid pressure, $D$ is the flexural rigidity of the ice, and $p$ is the externally imposed pressure.

Introducing the Fourier-Laplace transforms

$$
[\Phi, N, P]=\int_{-\infty}^{\infty} \mathrm{e}^{-\mathrm{i} k x} \mathrm{~d} x \int_{0}^{\infty} \mathrm{e}^{-s t}[\phi, \eta, p] \mathrm{d} t
$$

we obtain

$$
\begin{gathered}
\Phi_{y y}-k^{2} \Phi=0 \quad(-H<y<0), \\
\Phi_{y}=0 \quad(y=-H), \quad \Phi_{y}=s N \quad(y=0),
\end{gathered}
$$

and

$$
s \Phi+\left(g+D k^{4} / \rho\right) N=-P / \rho
$$

the solution of which yields

$$
\Phi=s N(k \sinh k H)^{-1} \cosh k(y+H),
$$

and

$$
N=-(P / \rho) k \tanh k H\left(s^{2}+k^{2} c^{2}\right)^{-1},
$$

where

$$
c^{2}(k)=\left(\frac{g}{k}+\frac{D k^{3}}{\rho}\right) \tanh k H
$$

Transforming the concentrated, moving load (1.1), we obtain

$$
P=F \int_{0}^{\infty} \exp [-s \tau-\mathrm{i} k X(\tau)] \mathrm{d} \tau,
$$

the substitution of which into (2.10), followed by the invocation of the convolution theorem for the inverse-Laplace transform of $P /\left(s^{2}+k^{2} c^{2}\right)$, yields (cf. (1.2))

$$
\eta(x, t)=-\frac{F}{2 \pi \rho} \int_{0}^{t} \mathrm{~d} \tau \int_{-\infty}^{\infty} \frac{\tanh k H}{c(k)} \exp \{\mathrm{i} k[x-X(\tau)]\} \sin [k c(t-\tau)] \mathrm{d} k .
$$


If $X(t)=V t$, the $\tau$ integral in (2.3) is elementary, and

$$
\eta(x, t)=-\frac{F}{4 \pi \rho} \int_{-\infty}^{\infty} \frac{\tanh k H}{k c(k)} \mathrm{e}^{\mathrm{i} k(x-V t)}\left[\frac{\mathrm{e}^{\mathrm{i} k(c+V) t}-1}{c+V}+\frac{\mathrm{e}^{\mathrm{i} k(V-c) t}-1}{c-V}\right] \mathrm{d} k,
$$

which is equivalent to Schulkes \& Sneyd (1988, equation (2.7)).

\section{Numerical results}

In order to obtain detailed results from (2.13) numerical methods are necessary. This equation can be written in the form

$$
\eta(x, t)=-\frac{F}{2 \pi \rho} \mathscr{F}_{k}^{-1} Q(k, t), \quad Q(k, t)=\frac{\tanh (k H)}{k c(k)} \int_{0}^{t} \mathrm{e}^{-\mathrm{i} k X(\tau)} \sin [k c(t-\tau)] \mathrm{d} \tau,
$$

where $\mathscr{F}_{k}^{-1}$ represents the inverse Fourier-transform operator with respect to the variable $k$. Numerical calculation of $Q(k, t)$ is not altogether straightforward. In computing the Fourier inverse a large range of $k$ values must be used, which requires the integration of rapidly oscillating functions. Special methods can be used, but here we consider the simpler case in which the acceleration (or deceleration) of the source is uniform. Then $Q(k, t)$ can be expressed in terms of Fresnel integrals as follows.

For an accelerating load $X=\frac{1}{2} A t^{2}$, where $A$ is a constant, the integral in (3.1) can be written in the form

where

$$
\frac{1}{2 \mathrm{i}}\left[I(c) \mathrm{e}^{\mathrm{i} c k t}-I(-c) \mathrm{e}^{-\mathrm{i} c k t}\right]
$$

$$
I(c)=(k / \beta) \exp \left(\mathrm{i} \gamma^{2}\right)[F((\beta(t+\alpha)))-F(\gamma)] .
$$

Here $\alpha=c / A, \gamma=\left(\frac{1}{2} k A\right)^{1 / 2}$, and the function $F(x)$ is defined by setting

$$
F(x)=\int_{0}^{x} \exp \left(\mathrm{i} t^{2}\right) \mathrm{d} t
$$

and can be defined in terms of Fresnel integrals,

$$
F(x)=\sqrt{\pi / 2}(C(x \sqrt{2 / \pi})-\mathrm{i} S(x \sqrt{2 / \pi}),
$$

in the notation of Abramowitz \& Stegun (1964). For the decelerating load $X(t)=V t-\frac{1}{2} A t^{2}$ we find

$$
I(c)=(k / \beta) \exp \left(\mathrm{i} \gamma^{2}\right)\left[F^{*}((\beta(t+\alpha)))-F^{*}(\gamma)\right],
$$

where the star denotes the complex conjugate, and now $\alpha=(V+c) / A$.

Figures $1(a)-1(d)$ show the time development of the wave system due to line loads moving with constant (or zero) acceleration. As in Schulkes \& Sneyd (1988) we present the results in the form of mesh plots of the surface elevation in the $(x, t)$ plane. The $x$-axis is placed in the immediate foreground, and the second horizontal axis represents time. In each case the surface is shown in a frame of reference moving with the load. We use dimensionless units in which the velocity, length and time scales are

$$
v_{0}=c_{\min }, \quad \eta_{0}=\frac{F}{\rho c_{\min }^{2}}, \quad t_{0}=\frac{\eta_{0}}{c_{\min }},
$$

respectively. Here $c_{\min }$ is the minimum phase velocity. For example in experiments carried out by Takizawa (1978) the ice thickness was $0.14 \mathrm{~m}$, with $c_{\min } \approx 5 \mathrm{~m} \mathrm{~s}^{-1}$. The 

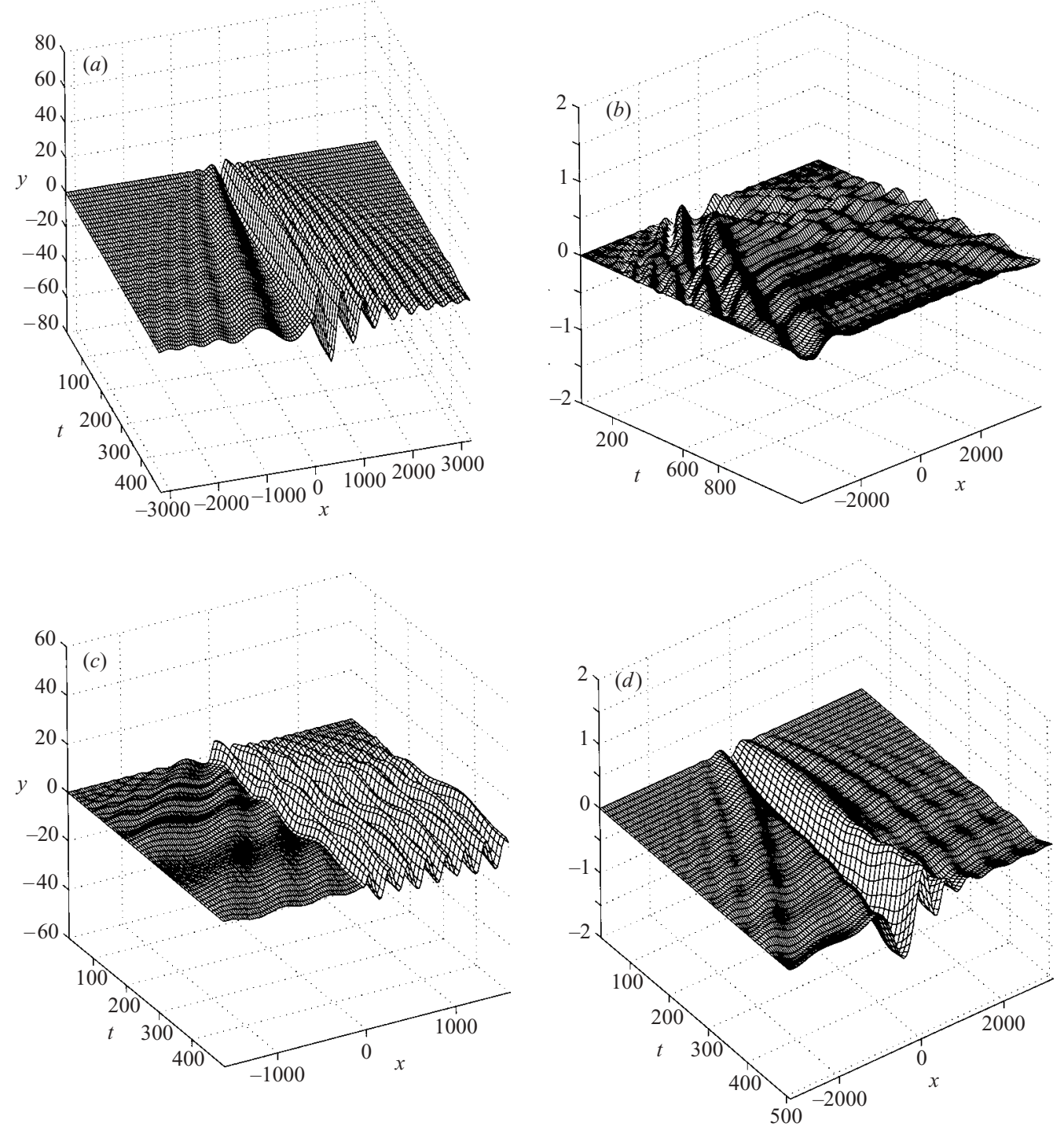

Figure 1. Time development of the wave system due to line loads: mesh plots of the surface elevation for $(a)$ constant subcritical velocity $v^{\prime}=0.4,(b)$ constant supercritical velocity $v^{\prime}=1.17,(c)$ constant acceleration 0.0784 to a final speed $v^{\prime}=2.0,(d)$ initial speed $v^{\prime}=0.4$, then constant deceleration to rest at time $t^{\prime}=1275$.

load was $350 \mathrm{~kg}$, and assuming a load width of $1 \mathrm{~m}$ the length and times scales would be $\eta_{0}=14 \mathrm{~cm}$ and $t_{0}=0.028 \mathrm{~s}$. A prime - for example $v^{\prime}-$ is used to denote the non-dimensionalized variables.

Figure 1(a) shows the wave pattern created by a load impulsively accelerated to a subcritical speed $v^{\prime}<1.0$. which then continues at constant speed. This is similar to figure 7 of Schulkes \& Sneyd (1988). Shorter, faster, waves propagate before the source, and slower, longer, waves behind. Figure $1(b)$ is similar, but now the speed is supercritical with $v^{\prime}>1.0$. Here the difference in wavelength between the forward and backward waves is more pronounced, as in figure 8 of Schulkes \& Sneyd (1988). 
Figure $1(c)$ shows the effect of a load starting from rest, then moving with a constant acceleration $a^{\prime}=0.0784$ to a maximum velocity of approximately $2 c_{\mathrm{cmin}}$. The most noticeable difference is that the wave amplitude increases more slowly because the load speed is increasing. In figure $1(d)$ the load accelerates impulsively to a speed $0.4 c_{\mathrm{cmin}} / 2$ then decelerates uniformly, coming to rest at time $t^{\prime}=1275$. At this point the waves have radiated away from the load, and the steady-state deflection begins to evolve.

\section{Conclusions}

In this article we have developed a method of calculating the deflection of a uniform floating ice sheet in response to a load moving at varying speed. Numerical Fourier-transform inversion can be used to visualize the ice-sheet deflection. We find no noticeable effect as a load accelerates through the critical speed $c_{\text {cmin }}$. This is to be expected since the displacement due to a load travelling at constant speed $c_{\text {cmin }}$ grows linearly with time (Schulkes \& Sneyd 1988). For loads moving with nonconstant acceleration special methods may be necessary to evaluate rapidly oscillating integrals.

This work was supported in part by the Office of Naval Research Grant N0001492-J-1171.

\section{REFERENCES}

Abramowitz, M. \& Stegun, J. 1964 Handbook of Mathematical Functions. Dover.

MiLeS, J. 1960 On the response of an elastic half-space to a moving blast wave. J. Appl. Mech. 27, $710-716$.

PǍrăU, E. \& Dias, F. 2002 Nonlinear effects in the response of a floating ice plate to a moving load. J. Fluid Mech. 460, 281-305.

Schulkes, R. M. S. M. \& SNEYD, A. D. 1988 Time-dependent response of floating ice to a steadily moving load. J. Fluid Mech. 186, 25-46.

Squire, V. A., Hosking, R. J., Kerr, A. D. \& Langhorne, P. S. 1996 Moving Loads on Ice Plates. Kluwer.

Takizawa, T. 1978 Deflection of a floating ice sheet subjected to a moving load II (in Japanese). Cold Regions Scie. Technol. 11, 171-180. 